\title{
Natural Orifice Translumenal Endoscopic Surgery with a miniature in vivo surgical robot
}

\author{
Amy C. Lehman · Jason Dumpert · Nathan A. Wood • \\ Abigail Q. Visty · Shane M. Farritor · Brandon Varnell • \\ Dmitry Oleynikov
}

Received: 11 April 2008/Accepted: 2 March 2009/Published online: 3 April 2009

(C) Springer Science+Business Media, LLC 2009

\begin{abstract}
Background The application of flexible endoscopy tools for Natural Orifice Translumenal Endoscopic Surgery (NOTES) is constrained due to limitations in dexterity, instrument insertion, navigation, visualization, and retraction. Miniature endolumenal robots can mitigate these constraints by providing a stable platform for visualization and dexterous manipulation. This video demonstrates the feasibility of using an endolumenal miniature robot to improve vision and to apply off-axis forces for task assistance in NOTES procedures.

Methods A two-armed miniature in vivo robot has been developed for NOTES. The robot is remotely controlled, has on-board cameras for guidance, and grasper and cautery end effectors for manipulation. Two basic configurations of the robot allow for flexibility during insertion and rigidity for visualization and tissue manipulation. Embedded magnets in the body of the robot and in an exterior surgical console are used for attaching the robot to the interior abdominal wall.
\end{abstract}

Presented at the 2008 Society of American Gastrointestinal and Endoscopic Surgeons (SAGES) Meeting, Philadelphia, Pennsylvania, April 9-12, 2008.

Electronic supplementary material The online version of this article (doi:10.1007/s00464-009-0451-1) contains supplementary material, which is available to authorized users.

A. C. Lehman · J. Dumpert - N. A. Wood .

A. Q. Visty · S. M. Farritor

N104 Walter Scott Engineering Center, University of Nebraska-

Lincoln, P.O. Box 880656, Lincoln, NE 68588-0656, USA

B. Varnell · D. Oleynikov $(\bowtie)$

983280 Nebraska Medical Center, University of Nebraska

Medical Center, Omaha, NE 68198-3280, USA

e-mail: doleynik@unmc.edu
This enables the surgeon to arbitrarily position the robot throughout a procedure.

Results The visualization and task assistance capabilities of the miniature robot were demonstrated in a nonsurvivable NOTES procedure in a porcine model. An endoscope was used to create a transgastric incision and advance an overtube into the peritoneal cavity. The robot was then inserted through the overtube and into the peritoneal cavity using an endoscope. The surgeon successfully used the robot to explore the peritoneum and perform small-bowel dissection.

Conclusion This study has demonstrated the feasibility of inserting an endolumenal robot per os. Once deployed, the robot provided visualization and dexterous capabilities from multiple orientations. Further miniaturization and increased dexterity will enhance future capabilities.

Keywords Natural orifice - In vivo - Robots - NOTES · Laparoscopy

Acknowledgments Oleynikov and Farritor are shareholders in the Virtual Incision Corporation, a startup company to commercialize this technology. 